\title{
Reactor Modelling and Analysis of Amorphous Hydrogenated Silicon Deposition by PECVD
}

\author{
A. Djelloul, B. Despax*, J.P. Couderc and P. Duverneuil \\ Laboratoire de Génie Chimique, URA 192 du CNRS, ENSIGC, Chemin de la Loge, 31078 Toulouse, \\ France \\ * Laboratoire de Génie Electrique, URA 304 du CNRS, Université Paul Sabatier, 118 Route de Narbonne, \\ 31062 Toulouse cedex, France
}

\begin{abstract}
The behaviour of plasma reactors is complex and affected by a large number of parameters (temperature, pressure, flow rates, power, frequency, etc...). In that context, modeling constitutes a very convenient theoretical approach to analyze the complex parameters influences on the reactors overall performances, in the particular case studicd here, amorphous hydrogenated silicon deposition rate profiles on the substrates.

This particular study is devoted to a detailed analysis of the reactor behaviour in higher electrical power conditions. It demonstrates that, if relatively simple mechanisms for electron-molecule interactions and gas phase reactions can be used in low power conditions, this do not remain true in higher power conditions where a great number of reactions must be taken into account.
\end{abstract}

\section{INTRODUCTION}

Thin hydrogenated amorphous silicon (a-SiH) films with convenient electronic properties can be produced by treatment of silane monomer in low-pressure RF discharges. The process, refereed to as plasma decomposition-deposition of silane, has been well studied for many years. However, the industrial use of this technology needs additional informations so as to increase reproducibility and to optimize the process. Plasma reactors are affected by a large number of parameters such as gas flow rate, temperature, reactor geometry, electrical power and frequency applied to the discharge. Modelling of reactors for plasma deposition constitutes, then, a very interesting tool to understand the relationship between these parameters and the reactor performances.

A first analysis in this way has been recently performed and published in our laboratory. It has provided a lot of interesting results on the basic three steps which must be taken into account, i.e. a) electron silane interactions b) gas phase chemistry and c) surface chemistry; however, this analysis used a relatively simple description of phenomena in the discharge and, as a consequence, was restricted to low electrical power conditions.

In fact, engineers are more interested in higher electrical power conditions, for evident reasons of reactors productivity and economy. This paper presents new results and new theoretical analysis performed in those more interesting but more difficult conditions.

\section{EXPERIMENTAL}

The main part of the equipment consists of a stainless-steel parallelipipedical plasma box located in a vacuum chamber (Figure -1-). The 13.56 MHz RF electrode is capacitively coupled to an RF generator by means of an LC matching network. Glass substrates $\left(20 \times 20 \mathrm{~cm}^{2}\right)$ can be clamped on the grounded electrode. The RF voltage applied to the driven electrode is controlled using an oscilloscope.

Silane gas at a pressure of 100 mTorr was used for the discharge. The whole reactor was uniformly heated at $500 \mathrm{~K}$. The experiments have been performed at different values of the RF voltage and discharge power, with a $40 \mathrm{sccm}$ gas flow rate. The thickness of the films deposited on the glass substrates were measured using a Tencor profiler. 


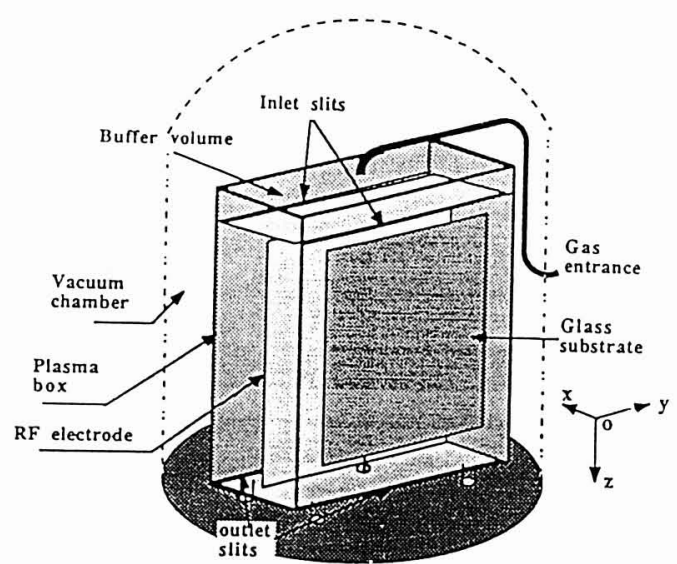

figure (1)

\section{MODELING}

\subsection{Volume Reactions, and Surface Reactions}

The model has been applied to the polyatomic gas $\mathrm{SiH}_{4}$ which upon dissociation in the plasma produces ions and neutral radicals such as $\mathrm{H}, \mathrm{SiH}_{2}, \mathrm{SiH}_{3} \ldots$. ... Although ions due to $\mathrm{SiH}_{4}$ electron interaction play an important role to determine the electrical behaviour of discharges, only neutral radicals constitute the dominant precursors to film growth. In agreement with previous studies on infrared laser absorption spectroscopy [1-2], we assumed that silyl $\left(\mathrm{SiH}_{3}\right)$ probably constitutes the dominant radical, in low power conditions when silane is pure. On the other hand, in the absence of definite informations on electron-silane cross sections for radical formation, it has been supposed, following Gallagher [3] that electron dissociation branching is similar to photodissociation branching [4]; as a consequence, silane dissociation produces, then, a dominant sylilene radical density, much more significant than the silyl radical density. These considerations and the use of the QRRK [5] (Quantum Rice-Ramsperger-Kassel) theory have allowed us to select three important reactions, suitable to constitute a first model involving a simplified chemical mechanism [5] (table - I-).

The surface reactions do not appear explicitly in the mass balance equation which will be presented in the following; they become effective only through boundary conditions. With the simplified chemical mechanism presented above, only $\mathrm{SiH}_{3}, \mathrm{SiH}_{2}$, and $\mathrm{H}$ can react on surfaces with, respectively, a surface loss probability $(B)$ of $0.26,0.8$ and $1[6]$. This coefficient $B$, is the sum of the sticking probability to the surface, which contributes to film growth (for example, for $\mathrm{SiH}_{3}, \mathrm{~s}=0,09$ ), and the recombination probability with hydrogen atoms producing $\mathrm{SiH}_{4}$ ( for example, for $\mathrm{SiH}_{3}, \gamma_{=0,17}$ ). The sticking coefficient of $\mathrm{SiH}_{2}$ has been taken equal to its loss probability ( $\mathrm{s}=\mathrm{B}$ ).

In a second step and as to increase the model quality, a more complex chemical mechanism has been taken into account, which considers interactions between electrons and disilane with two possible mechanisms

It is necessary, then, to consider several additional reactions which take into account the formation of higher order species $\left(\mathrm{Si} 3 \mathrm{H}_{8}, \mathrm{Si}_{4} \mathrm{H}_{10}, \ldots ..\right)$ (table -1-).

The first results obtained using this complex chemical mechanism have not been in good agreement with growth rate values measured experimentally at high discharge power. As a consequence it has been necessary to proceed further and to take into account the formation of $\mathrm{Si}$ and $\mathrm{SiH}$ radicals, by electronic impact dissociation of $\mathrm{SiH}_{4}$, and the contribution of these species to the film growth

The compete chemical mechanism involves, now, four electrons-silane interaction reactions and a large number of reactions in the gas phase [ 7] (table-1-). 


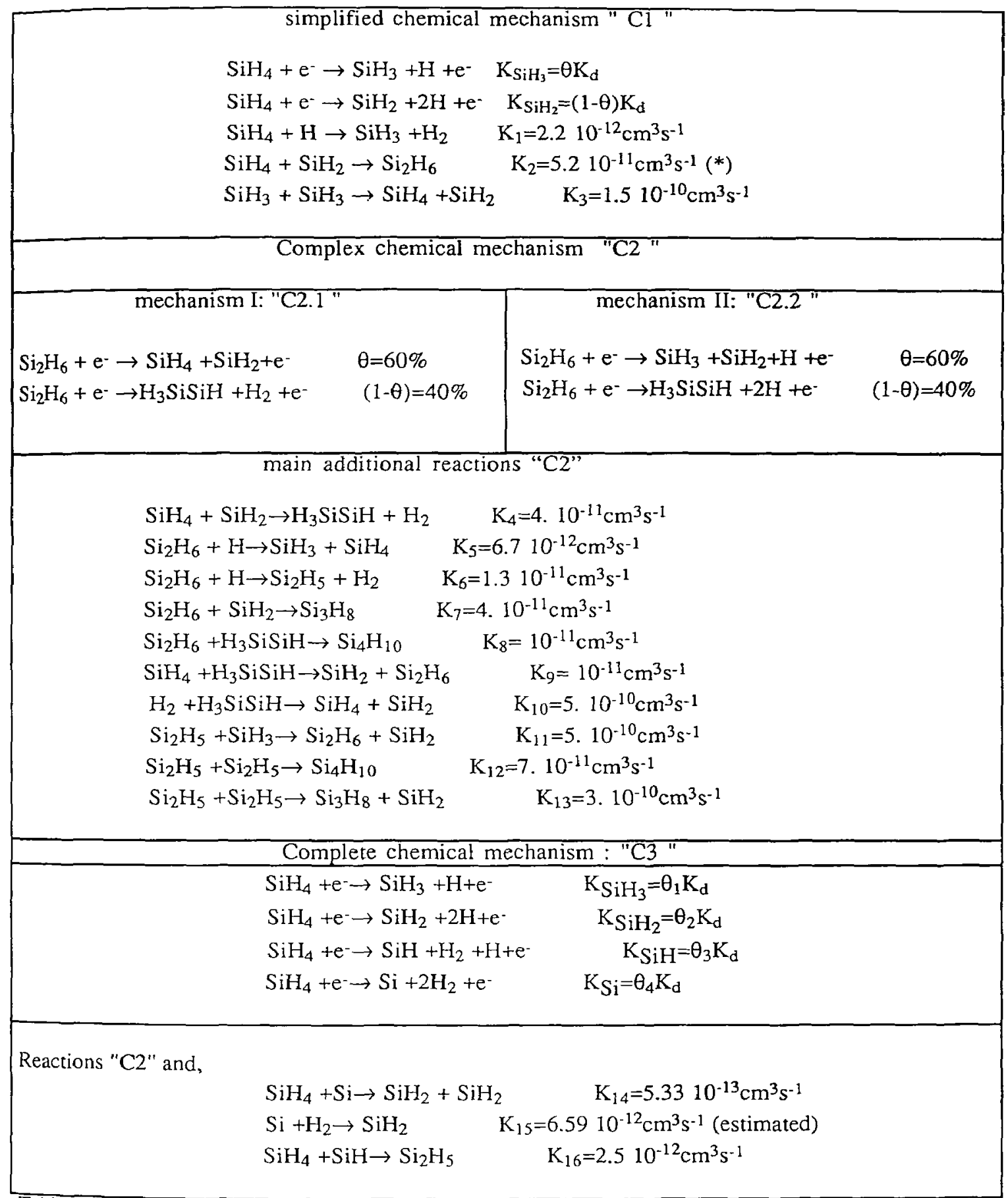

Table 1:Chemical schemes and kinetic data used at 0,1 Torr and $500 \mathrm{~K}$

* In complex mechanisms $\mathrm{k}_{2}=2.7510^{-11} \mathrm{~cm}^{3} / \mathrm{s}$

The silane dissociation constant $\left(\mathrm{K}_{\mathrm{d}}\right)$ is obtained by modelling of the electronic processes in the RF discharge by fluid model [8]. This procedure overestimating the electrical power and, thus silane 
dissociation [9] where the power dissipation predicted by the fluid model is about twice the experimental one ; the agreement between experiments and PIC-MC model is better, and when we compare the profil of the emission giveen by this model and fluid model we note that the SiH emission giveen by PIC-MC model is in agreement with the experimental results.[10]. As consequence it has been necessary that the dissociation constant has been divided by the ratio between the calculated and observed RF power, which are given by J.PERRIN[11] .

For the high values of the RF power, the silane dissociation constant, has been from the values obtained at lower power.

The branching coefficients $\left(\theta_{1}, \theta_{2}, \theta_{3}\right.$, and $\left.\theta_{4}\right)$ involved in the complete chemical mechanism, have been deduced from a detailed treatment of experimental results presented by PERRIN [ 6] which involves a complete modeling of its laboratory reactor and which will not be presented here in more details.

\subsection{General structure of the model}

Let us recall that L. LAYEILLON, in a recent important work, developed several model structures, starting from a complex two dimensional analysis then simplifying it progressively. This author concluded that a $2 \mathrm{x}$ 1D model, of the piston-diffusion type in the longitudinal direction for neutral species, coupled with a simple diffusion equation in the transversal direction, for radicals, probably constitutes the best compromise between precision and computing effort.

In this work, this relatively simple model structure has been selected.

Let us recall that the mass balance for neutral species is written as

with the following boundary conditions:

$$
V_{z} \frac{d N_{z}(k)}{d z}-D_{k} \frac{d^{2} N_{z}(k)}{d z^{2}}-R_{z}(k)=0
$$

at the entrance, $z=0, V_{z} N_{z}(k)-D_{k}\left(\frac{d N_{z}(k)}{d z}\right)=V_{z} N_{z}^{0}(k)$

at the exit, $\quad \mathrm{z}=\mathrm{L}, \frac{\mathrm{d}^{2} \mathrm{~N}_{\mathrm{z}}(\mathrm{k})}{\mathrm{dz}}=0$

For the radicals species, the equation is written as,

$$
D_{k} \frac{d^{2} N_{x}(k)}{d x^{2}}=R_{x}(k)
$$

At the wall, the consumption rate must be equal to the transport fluxes arriving on the surface, which results in the following boundary conditions:

on the left $, \quad x=0,-D_{k} \frac{d N_{x}(k)}{d x}=+R_{s}(k)$

on the right, $\quad x=L, \quad-D_{k} \frac{d N_{x}(k)}{d x}=-R_{s}(k)$

where

$$
R_{s}(k)=\beta_{k} \frac{V_{k}}{4} N_{x}(k), \quad V_{k}=\sqrt{\frac{8 k_{B} T}{\pi m_{k}}}
$$

We have also developed a perfectly mixed reactor model which constitutes the simplest way to describe the neutral particle behaviour in the reactor. It consists in considering that there does not exist any spatial variation of their composition:the zero gradient is a consequence of the rapid mixing of the species. Such an hypothesis can be used particularly for the reactors with small sizes and at low pressure, which seems convenient for our conditions of work.

The simplified balance equation is than written :

$$
F_{0}(k)-F(k)=R_{s}(k) \times S_{R}+R_{v}(k) \times v_{R}
$$

where $F(k), R_{V}(k), R_{S}(k)$ are respectively the molecular flow rate, the volume production (or consumption) rate, and the surface reaction rate for the $k^{\text {th }}$ species. The notations $s_{R}$ and $s_{V}$ represent respectively the reactor surface and its volume. 


\section{RESULTS}

\subsection{Experimental results}

To study the influence of RF voltage both on the growth rate and the silane consumption, we carried out several experiments for different values of the RF voltage, under the following conditions, $\mathrm{P}=100 \mathrm{mT}$ (orr, $Q=40 \mathrm{sccm}$ of pure silane, $\mathrm{T}=500 \mathrm{~K}$.

As can be seen on figure (2), the growth rate increase is closely related to the RF voltage increase. Figure (3) shows a uniform rate of deposition in the flow direction, except for the higher RF voltage value, for which we observe a significant increase of the deposition rate near the reactor outlet. This phenomena results from the formation of powder which pile up specially near the exit of the reactor. This effect modifies the discharge functioning by the apparition of electron attachment processes on the powder, which induce a rise of the consumed power density and then a preferential silane dissociation into this place.

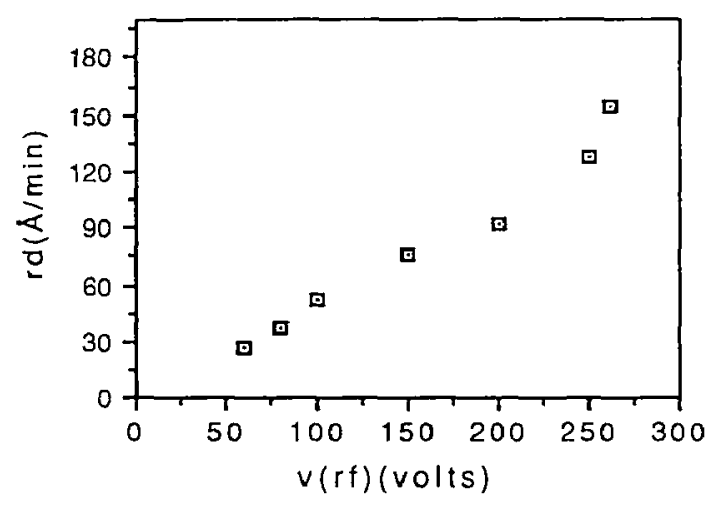

Fig (2): Experimental average growth rate for different values of RF voltage $(y=10 \mathrm{~cm})$

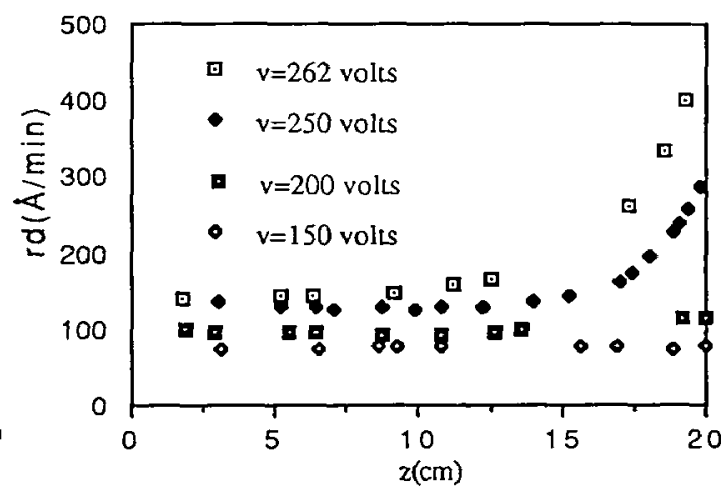

Fig(3) :Growth rate profile along the reactor for different values of RF voltage

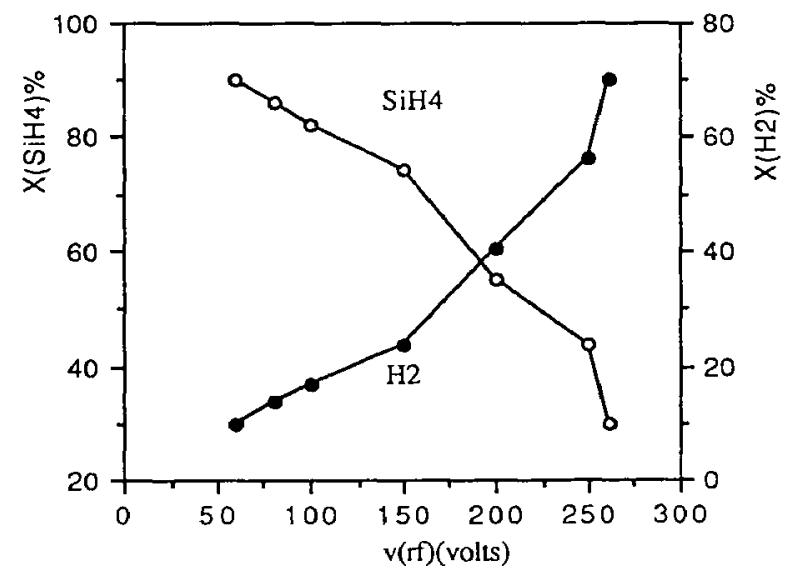

fig(4): Silane and hydrogen molar fraction obtained experimentally 
Gas composition at the exit of the reactor has been measured by mass spectrometry; then, silane depletion and hydrogen molar fraction have been calculated. The decrease of silane molar fraction when $\mathrm{RF}$ voltage increases is due to the increase of the dissociation rate which contributes to a direct consumption by electronic impact and an indirect consumption by radicals such as $\mathrm{SiH}_{2}$ and $\mathrm{H}$ issuing from electronic dissociation (figure -4- ).The gas mixture becomes richer and richer in molecular hydrogen (from $10 \%$ at 60 volts to $70 \%$ at 260 volts)

\subsection{Models results}

The first results we are going to analyze, now, have been obtained with the complex chemical mecharism "C2.2". The piston-diffusion reactor model allows to predict the evolution of the main stable species ( $\mathrm{SiH}_{4}$ and $\mathrm{H}_{2}$ ) along the flow direction (figure 5). It can be observed that the plasma reactor behaves quite as a perfectly mixed reactor, with an important concentration jump at the entrance of the reactor followed by a slow variation. The weakness of the concentration gradient must certainly be linked to the important contribution of diffusion under the tested condition $(P=100 \mathrm{~m}$ Torr, $\mathrm{T}=500 \mathrm{~K})$.

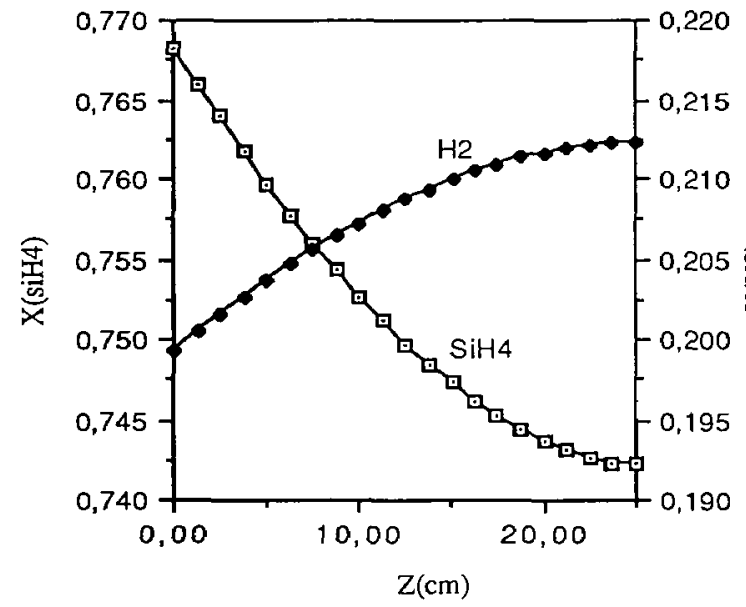

Fig (5) :Silane and hydrogen molar fraction from piston diffusion model with ' $\mathrm{C} 2.2$ ' at $\mathrm{V}(\mathrm{r} f)=150$ volts

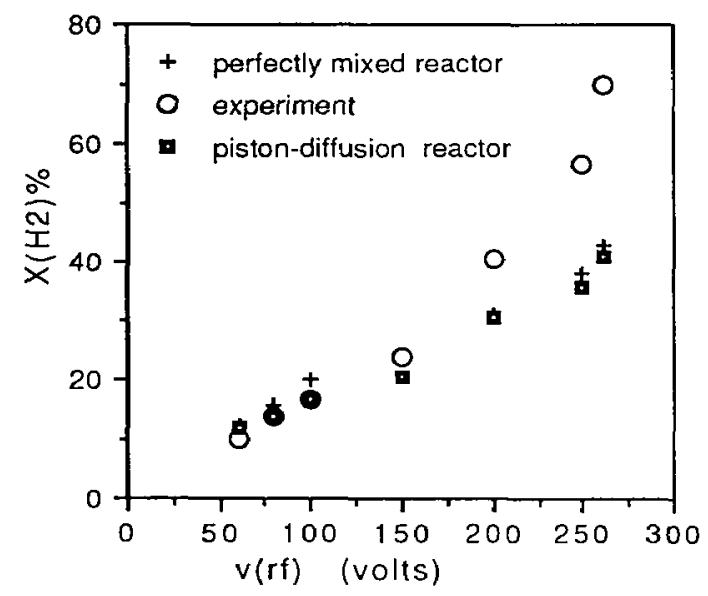

Fig (7) : Hydrogen molar fraction at the exit of reactor comparison between different models

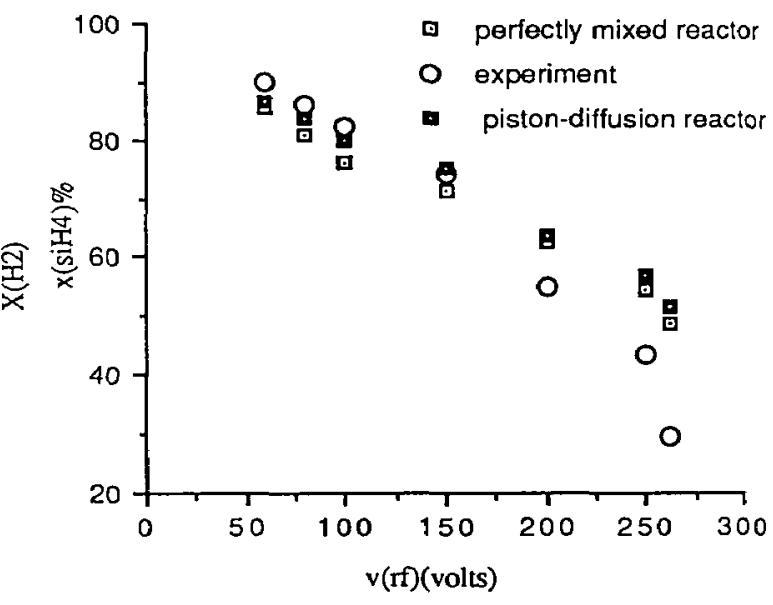

Fig (6) : Silane molar fraction at the exit of reactor, comparison between different models

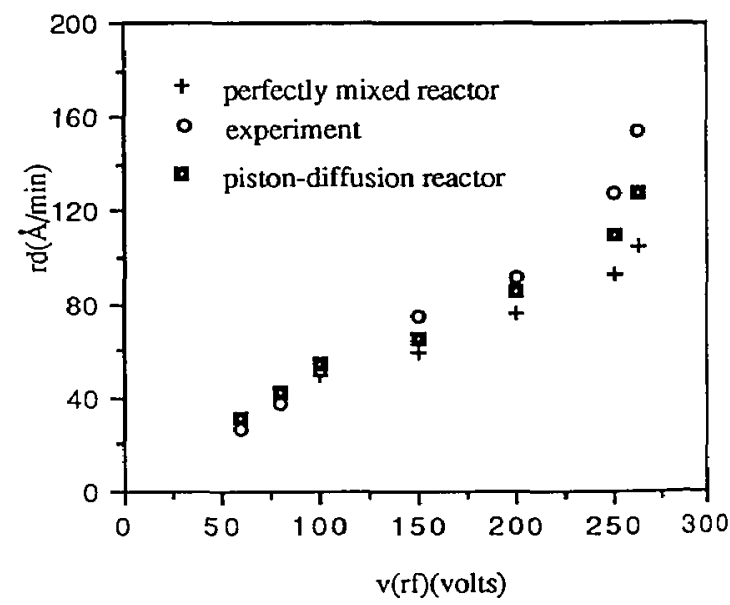

Fig (8): Average growth rate, comparison between different models $(y=10 \mathrm{~cm})$ 
As a logical consequence, figures (6-7) demonstrate that there is practically no difference between the results given by the piston-diffusion model and the perfectly mixed reactor model. However, the mean value of silane molar fraction in the piston diffusion model is slightly higher than the value in the perfectly mixed reactor.This slight difference in silane concentration leads to a difference in radical concentrations, and then, introduces a slight difference between the two models for the deposition rate (figure 8).

The discrepancy between the model and experimental results, for the higher values of the RF voltage, probably correspond to the choice of the complex chemical mechanism " $\mathrm{C} 2,2$ ", which do not take into account the formation of $\mathrm{SiH}$, and $\mathrm{Si}$ radicals by electrons-silane interactions and so neglect the contribution of these species to film growth.
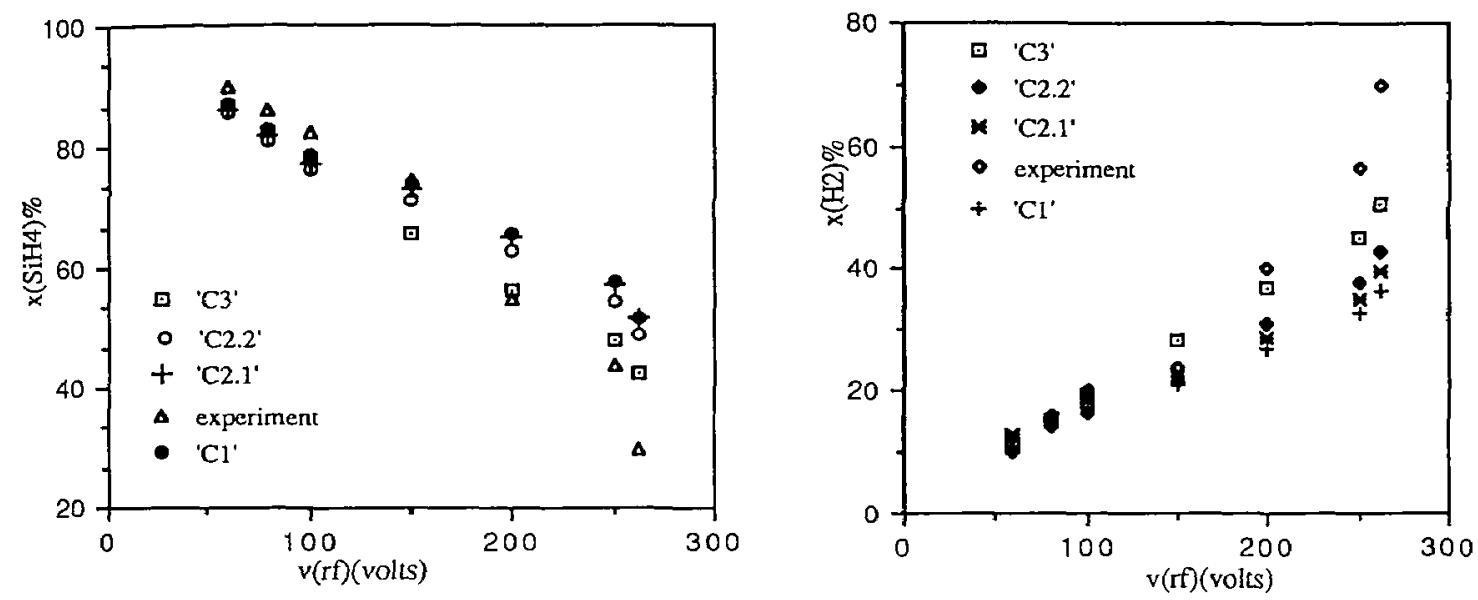

fig (9) : silane molar fraction from different chemical mechanism

fig (10): Hydrogen molar fraction from different chemical mechanism

In a second step, using the simpler perfectly mixed reactor model, a systematic analysis of influence of the chemical mechanism has been realized: differences appear for the higher RF voltage, as well for silane consumption as for hydrogen formation (figures 9 and 10).

Figure (11) shows that the exit molar fraction of disilane is strongly reduced with $\mathrm{C} 2$ and $\mathrm{C} 3$ chemistries in comparison with the simplified chemical mechanism where disilane is considered as inert. Finally and on the whole, the more complex mechanisms reach a convenient agreement with the experimental results.
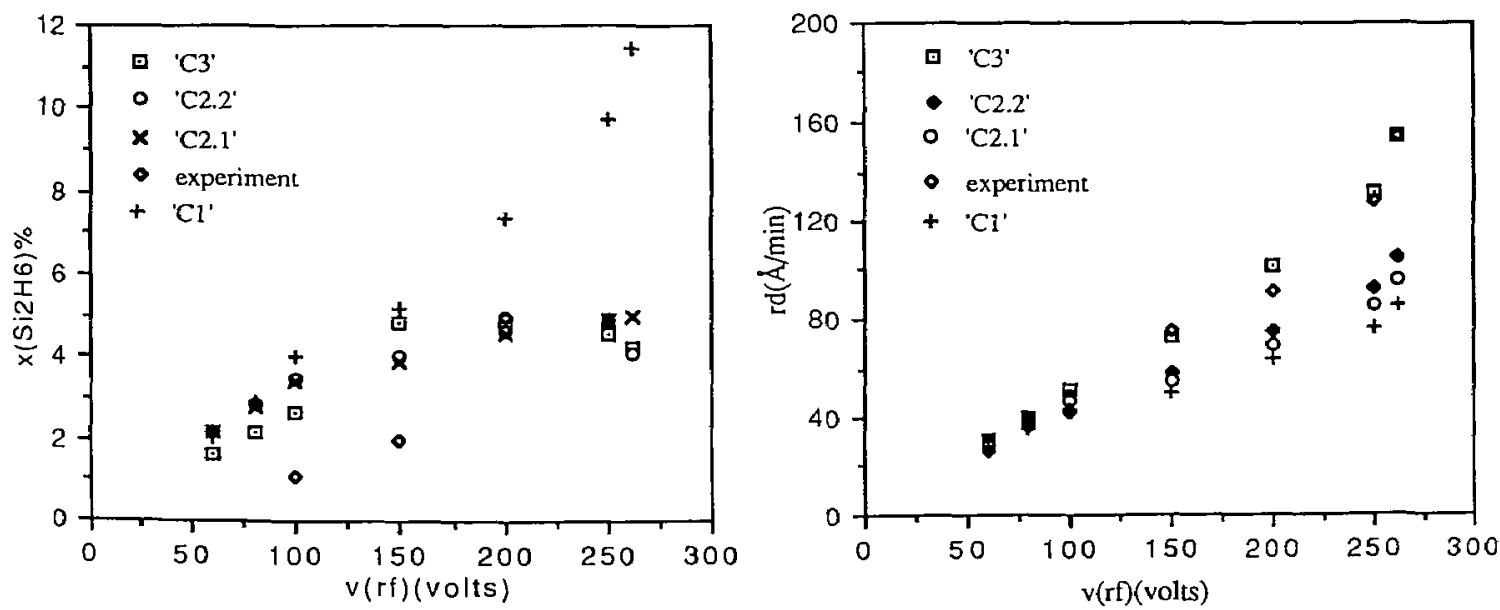

Fig (l 1): disilane molar fraction from different chemical mechanism

fig (12): Averge growth rate from different chemical mechanis $(y=10 \mathrm{~cm})$ 
Figure (12) which shows the deposition rate versus the RF voltage illustrates this good agreement.

The slight discrepancy which remains between the molar fractions of the main stable species $\left(\mathrm{S}_{i} \mathrm{H}_{4}, \mathrm{H}_{2}\right)$ obtained from the model and the experimental results could correspond to the difficulty to take into account the role played by electron attachment on powders. This attachment modifies the distribution function and consequently the dissociation rate by electron impact.

\section{CONCLUSIONS}

As soon as the reactor behaviour appeared to be quite perfectly similar to that of a perfectly mixed stage and as it became possible to use models relatively simple from a hydrodynamical point of view, attention could be turned towards the analysis of electronical and chemical phenomena.

This work has demonstrated that, if at low electrical power the discharge region involves simple chemical schemes, this remains by no way true at higher power. Several additional electron impact or chemical reactions must be considered to simulate conveniently experimental results.

However, it has been demonstrated that with complete electronical and chemical mechanisms, a quite convenient agreement between data and calculations can be reached, opening on a better understanding of the phenomena involved and probably on industrial applications improvements.

\section{REFERENCES}

[1] N.ITABASHI, K.KATO, N.NISHIWAKI, T.GOTO, C.YAMADA and E.HIROTA,Jap. J. Appl. Phys., Volume 27, 8, L1565 (1988)

[2] N.ITABASHI, K.KATO, N.NISHIWAKI, T.GOTO, C.YAMADA and E.HIROTA,Jap. J. Appl. Phys., Volume 28, 2 , L325 (1989)

[3] A.GAllagHER, J. Appl. Phys. Volume 63 (7), 2406 (1988)

[4] G.PERKINS, E.AUSTIN, and F.LAMPE, J. Am. Chem. Soc.Volume 101, 1109 (1979)

[5] L. LAYEILLON, Thèse de Doctorat, Institut National Polytechnique de Toulouse (1992)

[6] Patrick. KAE-NUNE, Jérôme PERRIN, Jean GUILLON, and Jacques JOLLY, to be published in Plasma Sources Sciences and technology'.

[7] Mark J. KUSHNER, J. Appl. Phys, Volume 63 (8) (1988)

[8] J. P. BOEUF, P. H. BELENGUER, L. C. PITCHFORD, 1. PERES, "Modélisation des décharge et des plasmas froids hors equilibre ", $8^{\text {th }}$ International Colloquium on plasma processes, Antibes (1991).

[9] Ph. BELENGUER, C. BÖHM, J.P. BOEUF, and J. PERRIN, proceedings of the XX $X^{\text {th }}$ Int. conf. on Phenomena in Ionized Gases, Pisa, Italy (1991).

[10] C. BÖHM, Thèse de doctorat, Laboratoire de Physique des Interfaces et des Couches Minces, Ecole Polytechnique de Palaiseau (1992).

[11] J. PERRIN, private communication 